\title{
Neuropsychological profile of hemiplegic migraines: a pediatric case study
}

\begin{abstract}
The clinical interview is an important part of a pediatric neuropsychological evaluation. Investigating the history of symptoms, the family, and genetic factors can often clarify etiological information. This manuscript will review a case of a 12 year old girl (HA) who has a family history of migraine headaches, but had previously been diagnosed and treated as a child with multiple mild grade 1 concussions. These suspected concussions were purportedly resulting in infrequent, but intermittent hemiplegic migraines. Specifically, HA suffered Hemiplegic Migraines which involved right dominant hand hemiplegia and significantly altered level of consciousness for approximately 12-24hours, resulting in a full return to physical and cognitive functioning post recovery. Medical records revealed practitioners did not explore family history or the sequence of events leading up to the migraines. There were even questions in the medical reports suggesting the hemiplegia may have reflected a conversion disorder. However, a thorough clinical interview including family history and neuropsychological, this case appears to show that HA has hemiplegia migraines along with neurocognitive impairments very consistent with previously published cases. Moreover her pattern of performance showed significant score variability and impairments on multiple largely left hemisphere and executive functioning tasks. Her specific cognitive impairments included a variety of left hemisphere cognitive skills (verbal comprehension, reading, bilateral executive deficits) as appears to suggest multifocal left hemisphere cerebral dysfunction.
\end{abstract}

Keywords: hemiplegic, neuropsychological, concussions, hemiplegia, encephalopathy
Volume I Issue 3 - 2014

\author{
Ashlee R Loughan,' Robert Perna, ${ }^{2}$ Jessica \\ Lee,' Jeremy Hertza' \\ 'NeuroBehavioral Associates, Augusta, Georgia, USA \\ ${ }^{2}$ TIRR Memorial Hermann, Houston, Texas, USA
}

Correspondence: Ashlee R Loughan, NeuroBehavioral Associates, 639 I3th Street, Augusta, Georgia, USA, Tel 207-7956I|0, Fax 207-5 |3-5| |6, Email aspicer88@yahoo.com

Received: June 12, 2014 | Published: July 02, 2014
Abbreviations: HM, hemiplegic migraine; FHM, familial hemiplegic migraine; SHM, sporadic hemiplegic migraine; BRIEF, behavior rating inventory of executive function; BASC-2, behavior assessment system for children- second edition; WISC-IV, wechsler intelligence scale for children-fourth edition; CVLT-C, california verbal learning test- children's version; CMS, children's memory scale; WIAT-III, wechsler individual achievement test-III; RCF, rey complex figure test; WCST, wisconsin card sorting test; CPT, continuous performance test; BASC, Behavior Assessment SelfReport; TOMM, test of memory malingering; MoA, migraine without aura

\section{Migraine}

Migraine is the second most common type of headache and a disabling brain disorder that consists of recurrent attacks of unilateral headaches that are often experienced in conjunction with nausea, phonophobia, and photophobia. ${ }^{1,2}$ Prior to the headache, one may experience an aura, or transient neurological symptoms, most often visual. The cumulative lifetime incidence of migraine is $43 \%$ in women and $18 \%$ in men $^{3}$ and is the leading cause for individuals to seek medical attention at neurological facilities. ${ }^{1}$ Prevalence estimates indicate that up to $10.6 \%$ of children ages 5 to 15 years and $28 \%$ of adolescents between the ages of 15 and 19years suffer with migraines. ${ }^{4}$ Despite its prevalence, migraine remains commonly undiagnosed or misdiagnosed, in both children and adults. ${ }^{5-7}$ In children, frequent headaches can cause a significant impact on educational functioning, ${ }^{8}$ as well as quality of life, prompting the need for early recognition and treatment. The long-term outcome of childhood headaches and evolution into adult headaches remains largely unknown.

The patho physiology of migraines is complex and is thought to be caused by changes in the brainstem and its interactions with the trigeminal nerve, a major pain pathway. Moreover, serotonin imbalance is thought to exacerbate and contribute to migraines. Stewart et al., ${ }^{3}$ suggests that the common occurrence of migraine may be linked to minor perturbations of normal brain function. Migraine pain begins with the activation of meningeal nociceptive trigeminal sensory afferents, which leads to are lease of vasoactive neuropeptides at their peripheral nerve terminals. Subsequently, there is an activation of second-order neurons in the trigeminal nucleus caudalis and then an activation of brain structures involved in the processing and perception of pain. ${ }^{2}$ The process of maintaining the severe prolonged pain of migraine headache is attributed to the sensitization of meningeal nociceptors and sensitization of central neurons of the trigemino vascular system. There is a strong genetic component but causative genes have not been identified, except for familial hemiplegic migraine.

\section{Hemiplegic migraine}

Hemiplegic migraine (HM) is a rare form of migraine with transient hemiplegia during the aura phase characterized by possible coma and prolonged neurological signs. ${ }^{9,10}$ There are approximately 100-200 families affected by hemiplegic migraine ${ }^{11}$ which have been published with two presentations: familial hemiplegic migraine (FHM) and sporadic hemiplegic migraine (SHM). Although no international studies have been completed, the prevalence of $\mathrm{HM}$ has been reported as $0.01 \%$, characterizing it as a very rare disease. ${ }^{12} \mathrm{An}$ epidemiological survey in Denmark was completed indicating the prevalence of sporadic form was at least $0.002 \%$ and of familial form was at least $0.003 \% .^{13,14}$ For FHM, patients have a positive family history and in SHM they do not. Hemiplegic migraine attacks in both FHM and SHM can be brought on by minor head trauma and are frequently associated with sensory disturbance such as paraesthesia or numbness, language difficulties, mild confusion, and visual 
disturbances such as scotoma, hemianopia, and blurred vision..$^{10,1}$ Some patients have experienced unconsciousness, coma, epileptic seizures, and diffuse encephalopathy. ${ }^{15,16}$ Typically, the neurological symptoms can last for an average of 60 minutes but they may last as long as five days in atypical attacks. In rare occasions, a diffuse encephalopathy, or migraine coma, may occur and confusion or stupor may be associated with pyrexia and cerebrospinal fluid pleocytosis. Attacks may begin in the first or second decades of life and decrease in frequency with age. ${ }^{9}$ Differential diagnoses include stroke, meningitis, and metabolic crisis that include mitochondrial disorders, non-accidental injury, acquired brain injury, and poisoning. ${ }^{10}$

\section{Familial hemiplegic migraine}

Familial hemiplegic migraine is a rare autosomal dominant subtype where at least one first- or second-degree relative also has migraine auras including motor weakness. These symptoms may last several weeks before resolving but some may not fully resolve, leading to a migraine infarction ${ }^{17}$ a stroke which occurs in the setting of a typical migraine attack. FHM can cause irreversible neurologic deficits, coma, epilepsy, intellectual disorder, and cerebellar atrophy. ${ }^{11,17}$ In terms of central neuronal processing, results have shown differences between FHM and common forms of migraine. FHM-1, one of the classifications of familial hemiplegic migraine, has many clinical similarities to migraine with (MA) and without aura (MoA), and an epidemiological study of a population-based FHM cohort shows that $65 \%$ of FHM patients had MA and/or MoA. Although MA and MoA are not associated with any of the known FHM mutations, there is a possibility of clinical similarities between FHM, MA, and MoA that would be rooted in common neurobiological pathways underlying the patho physiological mechanisms. ${ }^{18}$ Although genetic studies have implicated gene mutations, at least a quarter of these families do not have mutation in the genes presently identified suggesting the need for further research. ${ }^{11}$

\section{Sporadic hemiplegic migraine}

Sporadic hemiplegic migraine (SHM is defined as migraine attacks with some degree of motor weakness (hemiparesis) during the aura phase and where there is no first-degree relative with identical attacks. SHM appears to occur in isolation and is rarely associated with cerebellar abnormalities. The clinical symptoms accompanying SHM are identical to FHM but different from patients with migraine with typical aura. Patients with SHM do not have an increased risk of migraine without aura, but do have a higher increased risk of typical migraine with aura in comparison with the general population. ${ }^{10}$ Population-based studies have shown that SHM patients may have all four typical aura symptoms (visual, sensory, aphasic, and motor symptoms) and all have at least two of these aura symptoms during attacks. Additionally, De Sanctis et al. ${ }^{9}$ state that motor weakness is often unilateral and could involve both upper and lower limbs. About two-thirds of patients have symptoms that last only an hour or less, whereas in one-third of the cases, the duration of symptoms last longer than a day in about $8 \%$ of cases. ${ }^{9}$ A population search based prevalence study in Denmark found 105 patients with sporadic hemiplegic migraine at a prevalence rate of $0.01 \%{ }^{19}$

\section{Cognitive Profile}

\section{Migraines}

Although neuropsychological studies investigating cognitive functioning within the migraine population have reported mixed results, most studies evidence cognitive deficits when compared to controls. Brain based changes have been suggested including deep white matter lesions, cerebral hypo perfusion, reduced parietal and frontal gray matter, and cerebellar atrophy. ${ }^{20-25}$ Studies over the past few decades have revealed weaknesses in psychomotor ability, memory, processing speed, visual processing, attention, and executive functioning. ${ }^{1,26-33}$ Cognitive impairments are thought to be the result of either physiological dynamics preceding a migraine or of pronounced post-attack effects. However, other investigations and reviews have failed to demonstrate or conclude significant detrimental cognitive effects. ${ }^{34-36}$ Differences in findings have been attributed to patient selection bias, lack of distinction between groups, absence of control groups, and potential type I errors in the research. ${ }^{30}$

\section{Hemiplegic migraine}

Few studies have focused specifically on hemiplegic migraines and the potential impact on neuropsychological functioning. However, of the cases presented in the literature, it appears that cognitive deficits are not uncommon. Marchioni et al., ${ }^{37}$ presented a genetic case study of four first-degree relatives (ages 22-5yrs) affected by FHM in which all 4 individuals evidenced cognitive deficits across multiple domains including attention, language, memory, abstract thinking, reading, writing, and calculation abilities. Intellectual disability and ataxia were reported in 2 of 4 cases reported in a three-generational German FHM family. ${ }^{15}$ Additionally, according to Karner et al., ${ }^{21}$ there is a distinct pattern of preserved and impaired functioning in those challenged with hemiplegic migraines. Six family members (mean age 29.17 (12.62) age range 18-48yrs), all affected by FHM participated in neuropsychological assessments to determine cognitive profiling. All subjects had attacks since childhood (mean age of onset 5.22 (2.42)), frequently triggered by mild head trauma. Also, all demonstrated cerebellar dysfunction and oculomotor dysfunction with vertical and horizontal gaze evoked nystagmus. Four of 6 demonstrated cerebellar type dysarthria. MRI revealed atrophy especially in the vermal part of the cerebellum in all individuals. Neuropsychological assessment revealed preserved verbal intelligence, verbal memory, mental arithmetic, and general average linguistic ability (language comprehension and naming). Impairments were evidenced in figural memory (short \& long delay), executive functions, and attention (sustained and divided).

Unfortunately, there are few case presentations on children diagnosed with FHM which presents further limitations as research on adults may not generalize to the developing brain. Podesta et al,. ${ }^{38}$ reported on a 9 year-old boy in which neuropsychological testing (11 days post attack) revealed evidence "similar to a nonverbal learning disorder" (pg. 1500) and a related "discrepancy between Verbal $(\mathrm{SS}=99)$ and Performance IQ (SS=36)"(pg. 1498) with higher Verbal IQ. ${ }^{39}$ Imaging showed edema in the right cerebral hemisphere and EEG showed significant right hemispheric involvement. Repeated MRI showed a worsening of right hemispheric edema with mild deviation of septum pellucidum and compression of right lateral ventricle and third ventricle. Deficits were also seen in visual spatial analysis and integration, visual memory, digital agnosias, right-left orientation, and sustained and divided attention. Asghar et al,.$^{40}$ found acute cerebral edema on early imaging, but 1-4months afterwards, imaging and EEG were normal despite persisting cognitive impairments (i.e., attention, memory, and academic difficulties) in 3 children (ages 8,13 , and 15) with FHM. Most troubling is the evidence for significant symptom variability between the case presentation of a nine year old child who after age 2 had two reported HM attacks resulting in a severe regression in development, autistic demonstrations, and incomplete recovery remaining intellectually disabled $(\mathrm{IQ}=70$ at age 8$) .{ }^{41}$ 


\section{Case study}

The case of a 12year-old, right-handed Caucasian girl (HA), will be presented here. HA was referred for a neuropsychological evaluation to assess her cognitive profile following years of suspected multiple concussions and recorded hemiplegic migraines.

\section{Developmental/social information}

HA was born following a full-term (39weeks) and uncomplicated gestation and birth process. She weighed 9 pounds 6 ounces at birth. No gestational toxic exposure or complications during pregnancy were reported, though Apgar scores were not reported. At birth, HA was healthy with no documented medical complications. Verbal and motoric developmental milestones were reportedly met at age expected times including walking and talking. During childhood, HA was described as a healthy child. No history of ear infections, seizures, excessively high temperatures, or strokes was reported. HA currently lives with her biological parents and 3 brothers. She also has an older brother outside of the home setting. Parental education levels are both Bachelor Degrees. HA is currently in the $6^{\text {th }}$ grade with reported "average" grades, no special education services and no history of retention. Diet and sleep were reported as average. However, HA has a history of sleep walking (about once a year). Extended family history is positive for mental retardation, alcohol abuse, mental illness, depression/anxiety, and suicide attempts. Socially, HA was reported to be well adjusted and to have no difficulty getting along with her brothers and peers. She is involved in activities including basketball, softball, and swimming.

\section{Migraine history}

For the past 7years, HA has experienced multiple hemiplegic migraine episodes that were often precipitated by mild grade 1 concussions (up to 7) with the first incident at age 5. The concussions involved only transient alterations in consciousness suggestive of grade one concussions based on American Academy of Neurology guidelines..$^{42}$ Parental reports describe these incidents following any "bump" to the head, even if not appearing as a significant blow or traumatic from observation. Reported subsequent neurological symptoms of each episode included lethargy; numbness in the hands which travels through her arms to the back and up to the face resulting in right-sided hemiparesis; right side facial droop; slurred or reduced speech; extreme left-sided migraines (behind left eye); vomiting, loss of consciousness (duration 12-24hours); and blindness at times. Initially, it was believed that these symptoms were the results of her multiple concussions; and some records question a possible conversion disorder, however, this past year (6months prior to this evaluation), identical symptoms occurred following no reported head impact or trauma. This pattern and symptom base rates suggest that it is unlikely these symptoms were due to concussions, but there is no reported psychiatric history to warrant a conversion disorder diagnosis. When asked about triggers leading up to symptom onset, HA's parents explained that she usually becomes symptomatic immediately following physical exertion and parental reported of suspected dehydration. HA's parents were not initially forthcoming with information regarding the family history of migraines. After discussing how unusual her symptoms were to be considered possibly related to mild concussions, her parents finally mentioned the complex migraine history. HA's older brother has also reportedly experienced similar symptoms including vomiting and paralysis. HA's mother reports severe migraines without hemiparesis and her maternal grandfather was positive for a history of severe migraines since childhood ${ }^{43}$ (Figure 1).

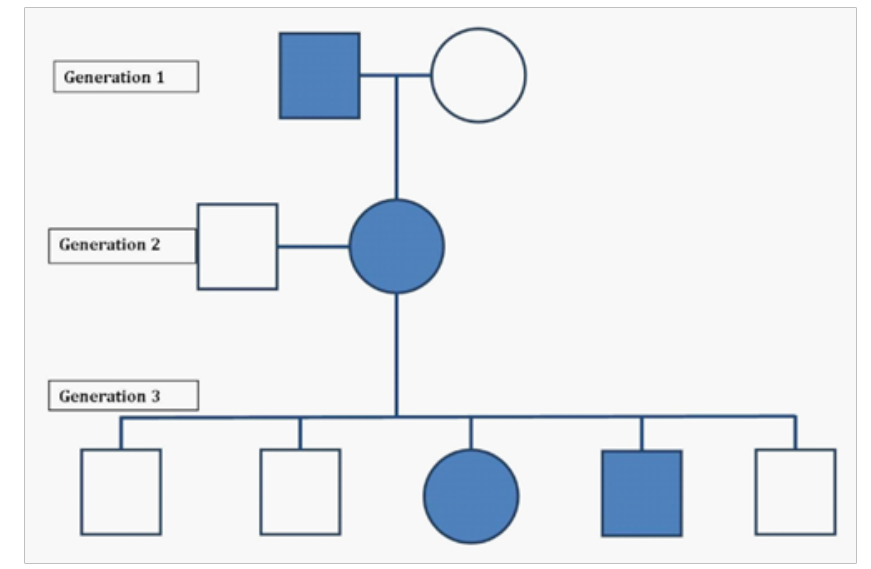

Figure I Family Genogram. The pedigree of this case's (HA's) family is shown Individuals with familial hemiplegic migraines are shown in solids. Circles are female and squares are male. Generation I= (left to right) Maternal Grandfather, Maternal Grandmother. Generation 2= (left to right) Father, Mother. Generation 3= (left to right) brother, brother, patient, brother, brother.

\section{Medical history}

Serial imaging was available for this case ranging from years 20042012. CT scans from 2004 and 2005 were normal. MRI images from 2010 and 2012 were normal. EEG (2012) revealed "severe left side cerebral dysfunction with severe cortical injury over left hemisphere or postictal left side seizure." Neuroimaging (SPECT/CT Scan; 2012) revealed findings of "decreased activity in the left temporal lobe, left occipital lobe, posterior left frontal lobe, left parietal lobe. There is more localized focally severe decreased activity in the left posterior inferior occipital lobe. There is crossed cerebellar diaschisis with relative decreased activity in the right cerebellum." Impressions reported "decreased left cortical brain activity most prominently in the left temporal, parietal, and focally in the posterior inferior left occipital lobe, consistent with left hemisphere diffuse axonal injury." Unfortunately, these scans were not available at the time of the evaluation. Medical record (2012) reported symptoms including concussion with LOC, epilepsy unspecified, syncope and collapse, migraine, and disturbance of skin. Impressions included the primary diagnosis of Migraine, hemiplegic. It should be noted that these 2012 medical records were performed within the days immediately following her most recent symptomatic event. To date, no genetic testing had been completed. No current medications were reported at this time.

\section{Reported current functioning}

When asked about current cognitive ability, HA reported average attention with limited distractibility. Overall, language was described as normal with occasional "fumbling of words." Receptive language was reported as average. Memory was reported as "good." No difficulty with vision or hearing was reported. When asked about emotional functioning, HA's parents reported no significant behavioral or emotional concerns. HA explained increased frustration with her younger siblings since her recent hospitalization (2011). Her parents agreed to a minimal decrease in tolerance and patience. However, no symptoms of sadness, anxiety, or anger were reported.

\section{Neuropsychological evaluation}

Evaluation procedures included administration, scoring and interpretation of comprehensive quantitative and qualitative 
measures, including the Behavior Rating Inventory of Executive Function (BRIEF); ${ }^{44}$ Behavior Assessment System for Children Second Edition (BASC-2); ${ }^{45}$ the Developmental and Behavioral History Questionnaire; and neuropsychological testing. HA was administered the Wechsler Intelligence Scale for Children-Fourth Edition (WISC-IV); ${ }^{46}$ California Verbal Learning Test - Children's Version (CVLT-C); ${ }^{47}$ Logical Memory subtest of the Children's Memory Scale (CMS);8 Verbal Fluency and Trails measures of the Delis-Kaplan Executive Function System (D-KEFS); ${ }^{49}$ the Wechsler Individual Achievement Test-III (WIAT-III); ${ }^{50}$ Rey Complex Figure Test (RCF) ${ }^{51}$ Wisconsin Card Sorting Test (WCST); ${ }^{52}$ Conners' Continuous Performance Test (CPT) ${ }^{53}$ Behavior Assessment SelfReport (BASC); ${ }^{45}$ and the Test Of Memory Malingering (TOMM). ${ }^{54}$

\section{Test results}

Neuropsychological results for HA are provided in (Table 1). Neuropsychological results were thought to be valid (TOMM=48 \& Reliable Digit Span=10). On the WISC-IV, HA obtained a borderline level of functioning Full Scale IQ (FSIQ=75). This Full Scale IQ appeared less than would be expected given her family and parental functioning and also given her pattern of scores across neurocognitive measures. Her profile demonstrated significant variability. A strength was noted in working memory ( $\mathrm{SS}=94)$ which fell in the average range and significantly above her Full Scale IQ. Verbal comprehension and processing speed were her weakest IQ scores, falling in the borderline range. Verbal Comprehension was significantly below working memory and amongst her lowest scores. This finding may be consistent with left hemisphere dysfunction.

In regard to attention, HA demonstrated both impulsivity and inattention to task. Divided attention was also impaired. Her basic expressive and receptive language appeared intact, with more difficulty when required to demonstrate understanding of language knowledge. When required to actively learn a word list, HAdemonstrated average to high average initial and delayed verbal learning. A similar, impressive trend of average scores was evidenced when presented verbal stories. Regarding visual memory HA was able to retain the majority of the details suggesting adequate visual memory. HA performed in the average range on a measure of phonetic ability, but in the low average range on measures of word reading. During mathematics, HA demonstrated low average math computation and reasoning. Overall, her academic ability across reading and mathematics appears to align or are above her overall cognitive ability and demonstrate no specific learning disabilities. HA was able to demonstrate average pattern recognition; however, her visual scanning and divided attention were impaired. Poor planning and reduced organization were also evident during visual motor tasks.

Table I Neuropsychological Evaluation Results

\begin{tabular}{|c|c|c|c|c|}
\hline \multirow[t]{2}{*}{ Measure } & \multirow[t]{2}{*}{ Index } & \multirow[t]{2}{*}{ Subtest } & \multicolumn{2}{|l|}{ HA's Profile } \\
\hline & & & Standard Score & Percentile \\
\hline \multirow[t]{15}{*}{ WISC-IV } & Full Scale & & 75 & 5 \\
\hline & & $\mathrm{VCl}$ & 75 & 5 \\
\hline & & Similarities & 80 & 9 \\
\hline & & Vocabulary & 85 & 16 \\
\hline & & Comprehension & 70 & 2 \\
\hline & & PRI & 82 & 12 \\
\hline & & Block Design & 95 & 37 \\
\hline & & Picture Concepts & 85 & 16 \\
\hline & & Matrix Reasoning & 75 & 5 \\
\hline & & WMI & 94 & 34 \\
\hline & & Digit Span & 90 & 25 \\
\hline & & Letter-Number Seq. & 100 & 50 \\
\hline & & PSI & 75 & 5 \\
\hline & & Coding & 70 & 2 \\
\hline & & Symbol Search & 85 & 16 \\
\hline \multicolumn{5}{|l|}{ WIAT-III } \\
\hline & \multicolumn{2}{|c|}{ Pseudo word Reading } & 96 & 39 \\
\hline & \multicolumn{2}{|l|}{ Reading Comp } & 81 & 10 \\
\hline & \multicolumn{2}{|l|}{ Spelling } & 91 & 27 \\
\hline & \multicolumn{2}{|l|}{ Numerical Op } & 76 & 5 \\
\hline & \multicolumn{2}{|c|}{ Math Problem Math } & 82 & 12 \\
\hline & \multicolumn{2}{|l|}{ Fluency } & 81 & 10 \\
\hline & & Addition & 71 & 3 \\
\hline & & Subtraction & 73 & 4 \\
\hline & & Multiplication & 84 & 14 \\
\hline \multicolumn{5}{|l|}{ DKEFS } \\
\hline & \multirow[t]{5}{*}{ Trails } & Visual Scanning & 70 & 2 \\
\hline & & Number Sequencing & 100 & 50 \\
\hline & & Letter Sequencing & 105 & 63 \\
\hline & & Number-Letter Switching & 60 & $<1$ \\
\hline & & Motor Speed & 110 & 75 \\
\hline & \multirow[t]{3}{*}{ Verbal Fluency } & Letter Fluency (correct) & 95 & 37 \\
\hline & & Category Fluency (correct) & 100 & 50 \\
\hline & & Category Switching (correct) & 75 & 5 \\
\hline
\end{tabular}


Table Continued...

\begin{tabular}{|c|c|c|c|c|}
\hline \multirow[t]{2}{*}{ Measure } & \multirow[t]{2}{*}{ Index } & \multirow[t]{2}{*}{ Subtest } & \multicolumn{2}{|l|}{ HA's Profile } \\
\hline & & & Standard Score & Percentile \\
\hline & & Category Switching (accuracy) & 80 & 9 \\
\hline \multicolumn{5}{|l|}{ WCST } \\
\hline & & Total Errors & 101 & 53 \\
\hline & & Perseverative Responses & 106 & 66 \\
\hline & & Categories Completed (raw) & 6 & $>16$ \\
\hline & & Learning to Lean (raw) & 0.26 & $>16$ \\
\hline \multicolumn{5}{|l|}{ CVLT-C } \\
\hline & & List A Trail I & 93 & 32 \\
\hline & & List A Trial 5 & 108 & 70 \\
\hline & & Recall Trials I-5 & 113 & 81 \\
\hline & & Short Delay Free Recall & 100 & 50 \\
\hline & & Short Delay Cued Recall & 108 & 70 \\
\hline & & Long Delay Free Recall & 115 & 84 \\
\hline & & Long Delay Cued Recall & 115 & 84 \\
\hline & & Recognition & 100 & 50 \\
\hline \multicolumn{5}{|l|}{ CMS } \\
\hline & Story Memory & Immediate & 100 & 50 \\
\hline & & Delay & 100 & 50 \\
\hline & & Recognition & 130 & 98 \\
\hline \multirow[t]{5}{*}{$\mathrm{CPT}$} & Omissions & & 108 & 70 \\
\hline & Commissions & & 123 & 94 \\
\hline & Hit RT & & 93 & 32 \\
\hline & Variability & & 123 & 94 \\
\hline & Perseverations & & 109 & 73 \\
\hline $\mathrm{RCF}$ & Copy & & 85 & 16 \\
\hline TOMM & Trial I (raw) & & 48 & \\
\hline
\end{tabular}

WISC-IV:Wechsler Intelligence Scale for Children-Fourth Edition; CVLT-C: California Verbal Learning Test-Children's Version; CMS: Children's Memory Scale; DKEFS: Delis-Kaplan Executive Function System; WIAT-III:Wechsler Individual Achievement Test-III; RCF: Rey Complex Figure Test; WCST: Wisconsin Card Sorting Test; CPT: Conners Continuous Performance Test;TOMM:Test of Memory Malingering

Overall, HA's cognitive profile suggests strength in her average basic working memory and low average visual reasoning. Similar scores were demonstrated during measures of expressive and receptive language, visual and verbal memory, reading, mathematics, and pattern recognition and visual sequencing. However, her test scores suggest below average ability in mental manipulation and cognitive flexibility (complex working memory), cognitive attention to task, divided attention, verbal comprehension, processing speed, and some executive deficits including planning, organization, and visual scanning. No overt emotional or behavioral difficulties were reported or observed.

\section{Discussion}

Though migraines are one of the leading causes for individuals to seek medical attention at neurological facilities ${ }^{55}$ their pathophysiology and the scope of their clinical presentation is not well understood. An uncommon migraine variant is hemiplegic migraine (HM) with prevalence rates reported to be $0.01 \%$. With similar neurologic symptoms as typical migraines, except for the additional hemiparesis symptoms, it would be assumed that HM would also present similar, if not more severe cognitive impairments. Few investigations have followed the cognitive profile of people with diagnosed HM, with even less documenting the full neuropsychological profile, specifically in pediatric cases.

Presented here was the case of a 12year-old, right handed Caucasian girl (HA) who presenting for her evaluation with the referral question concerning cognitive issues possibility secondary to concussions. Investigation of medical records and a thorough clinical interview supported the diagnoses of familial hemiplegic migraines. HA underwent a clinical interview and full neuropsychological evaluation. Although many of HA's cognitive abilities were low average or average; she did present areas of cognitive weakness compared to her peers. Her cognitive profile demonstrated deficits in verbal comprehension, reading, mental manipulation and cognitive flexibility (complex working memory), cognitive attention to task, divided attention, processing speed, and other executive deficits. Medical records showed that structural brain imaging (CT and MRI), results revealed no acute pathology and essentially normal images of HA's brain. However, functional imaging (SPECT) was suggestive of pathology. Specifically, the SPECT findings stated, "decreased left cortical brain activity most prominently in the left temporal, parietal, and focally in the posterior inferior left occipital lobe, consistent with left hemisphere diffuse axonal injury" This is consistent with some of HA's neuropsychological findings including weak overall verbal comprehension, reading, and executive deficits which are often bilateral (inattention, impulsivity, processing speed, and cognitive flexibility) and history of hemiparesis. These findings are suggestive of convergent evidence of neuropathology. However, it is plausible that the multiple concussions may also have contributed some to these impairments, though no acute concussion symptoms would be expected now that HA is 6months post her last concussion. Moreover, the history of hemiparesis cannot be attributed to mild sports concussions. Though somatoform disorder was considered by clinicians who did not fully explore the whole HM, there is no history of significant psychopathology, emotional trauma, or reason to suspect a conversion disorder, though consideration as a differential diagnosis may have appeared appropriate at some point. 
Based on the described concussions, HA appears to have a possible history of mild head trauma (up to 7 possible grade 1 concussions). Most such injuries result in full recovery ${ }^{56}$ and all conventional $\mathrm{CT}$ and MRI imaging results were normal (as would be expected following mild concussions), though more advanced modalities suggest potential micro structural and functional neurobiological changes. ${ }^{56,57}$ Multiple concussions in an individual further complicate this issue, particularly when considering the dynamic nature of the developing adolescent brain $^{58,59} \mathrm{Up}$ to 3 months after concussion children ages 8 to 16 have been found to have persistent deficits in processing complex visual stimuli ${ }^{60,61}$ but few other persistent residual functions have been consistently found. Other research suggesting possible residual symptoms is studies showing that children with 2 or more concussions tend to have lower grade point averages. ${ }^{57}$ Based on the aforementioned issues, there is some possibility that HA's cognitive profile may reflect some mild concussion residual; however, her weaker language skills are consistent with functional imaging (SPECT) and her episode of migraine related hemiplegia.

What is important to note in the presentation of this case to the literature is that without the in-depth clinical interview, investigation into the HA's medical history, functional imaging records, and query of her family and genetic history; this diagnosis and resulting cognitive impairments may have been misattributed to psychological factors or cumulative concussions. Dooley et al,. ${ }^{62}$ promote the utility of thorough clinical histories given their results showing that $93 \%$ of final diagnoses were reached after completing the clinical history, rather than $66 \%$ from the referral letter and only $6 \%$ influenced by the physical examination. As mentioned previously, HA presented as a child who was suspected of having multiple concussions with some possible mild residual cognitive issues. This testing could have easily been completed without any mention or exploration into the additional neurologic symptoms leading to genetic and brain based inquisitions. Thus, never revealing or understanding the true etiology of HA's neurologic condition: Hemiplegic Migraines. Research reveals that frequently, FHM is triggered by mild head trauma $^{10,21}$ which appears to be the case in most of HA's episodes. It was the thorough clinical interview and research into the suspected genetic link (FHM) which divulged the required information for the appropriate conceptualization of this rare and specific neurologic case presentation instead of misdiagnosing the etiology of deficits as multiple concussions. This highlights the value of a thorough clinical interview and aneuro psychological evaluation if appropriate. Moreover, given the multiple reoccurrence of HA's symptoms and current objectively documented residual cognitive symptoms, HA's care providers will now likely re-evaluate the need for prophylaxis medication to reduce the risk of future episodes.

\section{Limitations}

Though this case highlights a rare disorder and the benefits of thorough history taking, case studies only describe one person's clinical presentation. The addition of more family members or additional children diagnosed with FHM would add to the literature and overall clinical profile of children challenged by FHM. It will be important to continue to monitor and assess this child (serial testing) to determine if changes are occurring in her neurocognitive profile through time and possible further incidents.

\section{Acknowledgments}

None.

\section{Conflicts of interest}

Author declares there are no conflicts of interest.

\section{Funding}

None.

\section{References}

1. de Araujo CM, Barbosa IG, Lemos SM A, et al. Cognitive impairment in migraine. Dement Neuropsychol . 2012;6(2):74-79.

2. Pietrobon D. Insights into migraine mechanisms and CaV2.1 calcium channel function from mouse models of familial hemiplegic migraine. $J$ Physiol. 2010;588(Pt 11):1871-1878.

3. Stewart WF, Wood C, Reed ML, et al. Cumulative lifetime migraine incidence in women and men. Cephalalgia. 2008;28(11):1170-1178.

4. Powers SW, Patton SR, Hommel KA, et al. Quality of life in childhood migraines: Clinical impact and comparison to other chronic illnesses. Pediatrics. 2003;112(Pt 1):e1-e5.

5. Diamond ML. The role of concomitant headache types and non-headache co-morbidities in the under diagnosis of migraine. Neurology.2002;58(9 Suppl 6):S3-S9.

6. Hershey AD, Winner PK. Pediatric migraine: recognition and treatment. J Am Osteopath Assoc. 2005;105(4 Suppl 1):2S-8S.

7. Yi X, Cook AJ, Hamill-Ruth RJ, et al. Cervicogenic headache in patients with presumed migraine: missed diagnosis or misdiagnosis? J Pain. 2005;6(10):700-703.

8. Hershey AD1, Powers SW, Vockell AL, et al. PedMIDAS: development of a questionnaire to assess disability of migraines in children. Neurology. 2001;57(11):2034-2039.

9. De Sanctis S1, Grieco GS, Breda L, et al. Prolonged sporadic hemiplegic migraine associated with a novel de novo missense ATP1A2 gene mutation. Headache: Headache. 2011;51(3):447-450.

10. Hart AR, Trinick R, Connolly DJ, et al. Profound encephalopathy with complete recovery in three children with familial hemiplegic migraine. J Paediatr Child Health. 2009;45(3):154-157.

11. Russell MB, Ducros A. Sporadic and familial hemiplegic migraine: pathophysiological mechanisms, clinical characteristics, diagnosis, and management. Lancet Neurol. 2011;10(5):457-470.

12. Hansen JM, Schytz HW, Larsen VA, et al. Hemiplegic migraine aura begins with cerebral hypoperfusion: imaging in the acute phase. Headache. 2011;51(8):1289-1296.

13. Thomsen LL, Eriksen MK, Roemer SF, et al. A population-based study of familial hemiplegic migraine suggests revised diagnostic criteria. Brain . 2002;125(Pt 6):1379-1391.

14. Thomsen LL, Ostergaard E, Olesen J, et al. Evidence for a separate type of migraine with aura: sporadic hemiplegic migraine. Neurology . 2003;60(4):595-601.

15. Freilinger T, Bohe M, Wegener B, et al. Expansion of the phenotypic spectrum of the CACNA1A T666M mutation: a family with familial hemiplegic migraine type 1 , cerebellar atrophy and mental retardation. Cephalalgia . 2008;28(4):403-407.

16. Takahashi T, Arai N, Shimamura M, et al. Autopsy case of acute encephalopathy linked to familial hemiplegic migraine with cerebellar atrophy and mental retardation. Neuropathology. 2005;25(3):228-234.

17. Black DF, Kung S, Sola CL, et al. Familial hemiplegic migraine, neuropsychiatric symptoms, and Erdheim-Chester disease. Headache. 2004;44(9):911-915.

18. Hansen JM, Thomsen LL, Olesen J, et al. Familial hemiplegic migraine type 1 shows no hypersensitivity to nitric oxide. Cephalalgia. 2008;28(5):496-505.

19. Bhatia R, Desai S, Tripathi M, et al. Sporadic hemiplegic migraine: Report of a case with clinical and radiological features. $J$ Headache Pain. 2008;9(6):385-388. 
20. Calandre EP, Bembibre J, Arnedo ML, et al. Cognitive disturbances and regional cerebral blood flow abnormalities in migraine patients: their relationship with the clinical manifestations of the illness. Cephalalgia. 2002;22(4):291-302.

21. Karner E, Delazer M, Benke T, et al. Cognitive functions emotional behavior and quality of life in familial hemiplegic migraine. Cogn Behav Neurol . 2010;23(2):106-111.

22. Kurth T, Mohamed S, Maillard P, et al. Headache, migraine, and structural brain lesions and function: population based epidemiology of vascular ageing-MRI study. BMJ. 2011;342:c7357.

23. Martins IP, Cunha e Sa M. Loss of topographic memory and prosopagnosia during migraine aura. Cephalalgia. 1999;19(9):841-843.

24. Porter A, Gladstone JP, Dodick DW. Migraine and white matter hypertensities. Curr Pain Headache Rep. 2005;9(4):289-293.

25. Schmitz N, Arkink EB, Mulder M, et al. Frontal lobe structures and executive function in migraine patients. Neurosci Lett. 2008;440(2):92-96.

26. Chronicle E, Mulleners W. Might migraine damage the brain? Cephalalgia. 1994;14(6):415-418.

27. Chronicle EP, Wilkins AJ, Coleston DM. Thresholds for detection of a target against background grating suggests visual dysfunction in migraine with aura but not migraine without aura. Chephalalgia. 1995;15(2):117-122.

28. Coleston DM, Chronicle E, Ruddock KH, et al. Precortical dysfunction of spatial and temporal visual processing in migraine. J Neurol Neurosurg Psychiatry. 1994;57(10):1208-1211.

29. Hooker WD, Raskin NH. Neuropsychological alterations in classic and common migraine. Arch Neurol. 1986;43(7):709-712.

30. Mulder EJ, Linssen WH, Passchier J, et al. Interictal and postictal cognitive changes in migraine. Cephalalgia. 1999;19(6):557-565.

31. Schoenen J. Beta blockers and the central nervous system. Cephalalgia. 1986;6(Suppl 5):47-54

32. Wray SH, Mijovic-Prelec D, Kosslyn SM. Visual processing in migraineurs. Brain. 1995;118(Pt 1):25-35.

33. Zeitlin C, Oddy M. Cognitive impairment in patients with severe migraine. Br J Clin Psychol. 1984;23(Pt 1):27-35.

34. Burker E, Hannay HJ, Halsey JH. Neuropsychological functioning and personality characteristics of migrainous and non migrainous female college students. Neuropsychology. 1989;3(2):61-73.

35. Leijdekkers ML, Passchier J, Goudswaard P, et al. Migraine patients cognitively impaired? Headache. 1990;30(6):352-358.

36. Suhr JA, Seng EK. Neuropsychological functioning in migraine: Clinical and research implications. Cephalalgia. 2012;32(1):39-54.

37. Marchioni E, Galimberti CA, Soragna D, et al. Familial hemiplegic migraine versus migraine with prolonged aura: An uncertain diagnosis in a family report. Neurology. 1995;45(1):33-37.

38. Podesta B, Briatore E, Boghi A, et al. Transient nonverbal learning disorder in a child suffering from Familial Hemiplegic Mirgraine. Cephalalgia. 2011;31(14):1497-1502.

39. Wechsler D. Wechsler Intelligence Scale for Children. (3rd edn), Siunti OS, Italy. 2006

40. Asghar SJ, Milesi-Halle A, Kaushik C, et al.Variable manifestations of familial hemiplegic migraine associated with reversible cerebral edema in children. Pediatr Neurol . 2012;47(3):201-204.

41. Vanmolkot KR, Stroink H, Koenderink JB, et al. Severe episodic neurological deficits and permanent mental retardation in a child with novel FHM2 ATP1A2 mutation. Ann Neurol. 2006;59(2):310-314.
42. Giza CC, Kutcher JS, Ashwal S, et al. Summary of evidence based guideline update: Evaluation and management of concussion in sports: Report of the guideline development Subcommittee of the American Academy of Neurology. Neurology. 2013;80(24):2250-2257.

43. Lebas A, Guyant-Marechal L, Hannequin D, et al. Severe attacks of familial hemiplegic migraine, childhood epilepsy and ATP1A2 mutation. Cephalalgia. 2008;28(7):774-777.

44. Gioia GA, Isquith PK, Guy SC, et al. Behavior Rating Inventory of Executive Function. Child Neuropsychol. 2000;6(3):235-238

45. Reynolds CR, Kamphaus RW.The Clinician's Guide to the Behavior Assessment System for Children (BASC). Guilford Press, New York, USA. 2002

46. Wechsler D. Wechsler Intelligence Scale for Children. (4th edn), The Psychological Corporation, San Antonio, Texas, USA. 2003.

47. Delis DC, KramerJH, Kaplan E, et al. California Verbal Learning TestChildren's Version. The Psychological Corporation, San Antonio, Texas, USA. 1994.

48. Cohen B. Children's Memory Scale. The Psychological Corporation. San Antonio, Texas, USA. 1997.

49. Delis DC, Kaplan E, Kramer JH. The Delis-Kaplan Executive Function System. The Psychological Corporation, San Antonio, Texas, USA. 2001.

50. Wechsler D. Wechsler Individual Achievement Test (WIAT-III). (3rd edn), Pearson, San Antonio, Texas, USA. 2009

51. Meyers JE, Meyers KR. Rey Complex Figure Test and Recognition Trial. Psychological Assessment Resources Inc., Odessa, Florida, USA. 1996.

52. Heaton RK, Chelune GJ, Talley JL, et al. Wisconsin Card Sorting Test manual: Revised and expanded. Psychological Assessment Resources Inc., Odessa, Florida, USA. 1993.

53. Conners CK, Epstein JN, Angold A, et al. Continuous performance test performance in a normative epidemiological sample. J Abnorm Child Psychol. 2003;31(5):555-562.

54. Tombaugh TN. TOMM,Test of Memory Malingering, Multi-Health Systems Inc., New York, USA. 1996.

55. Lipton RB, Stewart WF, Diamond S, et al. Prevalence and burden of migraine in the United States: Data from the American Migraine Study II. Headache. 2001;41(7):646-657.

56. Choe MC, Babikian T, DiFiori J, et al. A pediatric perspective on concussion pathophysiology. Curr Opin Pediatr . 2012;24(6):689-695.

57. Halstead ME, Walter K. Sports related concussion in children and adolescents. Pediatrics. 2010;126(3):597-615.

58. Gioia GA. Pediatric assessment and management of concussions Pediatr Ann. 2012;41(5):198-203.

59. Reddy CC, Collins MW, Gioia GA. Adolescents sport concussion. Phys Med Rehabil Clin N Am . 2008;19(2):247-269.

60. Moser RS, Schatz P, Jordan BD. Prolonged effects of concussion in high school athletes.Neurosurgery. 2005;57(2):300-306.

61. Brosseau-Lachaine O, Gagnon I, Forget R, et al. Mild traumatic brain injury induces prolonged visual processing deficits in children.Brain Inj . 2008;22(9):657-668.

62. Dooley JM, Gordon KE, Wood EP,et al. The utility of the physical examination and investigations in the pediatric neurology consultation. Pediatr Neurol. 2003;28(2):96-99. 